Acta Crystallographica Section E

Structure Reports

Online

ISSN 1600-5368

\section{Low-temperature redetermination of tribenzylchloridotin(IV)}

\section{Seik Weng Ng}

Department of Chemistry, University of Malaya, 50603 Kuala Lumpur, Malaysia Correspondence e-mail: seikweng@um.edu.my

Received 20 January 2009; accepted 23 January 2009

Key indicators: single-crystal X-ray study; $T=100 \mathrm{~K}$; mean $\sigma(\mathrm{C}-\mathrm{C})=0.005 \AA$; $R$ factor $=0.026 ; w R$ factor $=0.074 ;$ data-to-parameter ratio $=19.7$.

Compared to the previous studies [Ng (1997). Acta Cryst. C53, 56-58; Yin et al. (2005). Huaxue Shiji, 27, 295-296], the redetermined structure of the title compound, $\left[\mathrm{Sn}\left(\mathrm{C}_{7} \mathrm{H}_{7}\right)_{3} \mathrm{Cl}\right]$, exhibits a doubled $c$ unit-cell parameter. There are two molecules in the asymmetric unit, with both $\mathrm{Sn}$ and both $\mathrm{Cl}$ atoms having 3 site symmetry. The $\mathrm{Sn}$ atoms have distorted $\mathrm{SnClC}_{3}$ tetrahedral geometries and the molecules interact by way of short $\mathrm{Sn} \cdots \mathrm{Cl}$ bridges $[\mathrm{Sn} \cdots \mathrm{Cl}=3.418(2)$ and 3.475 (2) $\AA$ ], thereby forming chains propagating in $c$.

\section{Related literature}

For the room-temperature structure of the title compound described in the $R 3$ space group but with the unique $c$ axis half as long, see: $\mathrm{Ng}$ (1997); Yin et al. (2005). For the direct synthesis of the title compound from metallic tin and benzyl chloride, see: Sisido et al. (1961).

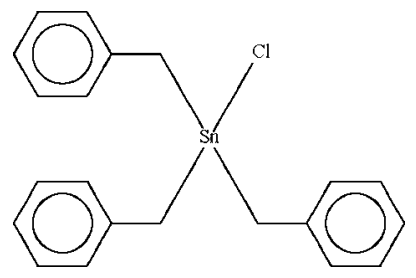

\section{Experimental}

Crystal data

$\left[\mathrm{Sn}\left(\mathrm{C}_{7} \mathrm{H}_{7}\right)_{3} \mathrm{Cl}\right]$

$M_{r}=427.52$

Trigonal, $R 3$

$a=16.7985$ (2) ̊

$c=11.6875$ (2) $\AA$

$V=2856.23(6) \AA^{3}$

Data collection

Bruker SMART APEX

diffractometer

Absorption correction: multi-scan

(SADABS; Sheldrick, 1996)

$T_{\min }=0.589, T_{\max }=0.917$

Refinement

$R\left[F^{2}>2 \sigma\left(F^{2}\right)\right]=0.026$

$w R\left(F^{2}\right)=0.074$

$S=1.07$

2737 reflections

139 parameters

1 restraint

$Z=6$

Mo $K \alpha$ radiation

$\mu=1.48 \mathrm{~mm}^{-1}$

$T=100$ (2) K

$0.40 \times 0.08 \times 0.06 \mathrm{~mm}$

9077 measured reflections 2737 independent reflections 2431 reflections with $I>2 \sigma(I)$

$R_{\text {int }}=0.014$

Table 1

Selected bond lengths (Å).

\begin{tabular}{llll}
\hline Sn1-C1 & $2.146(3)$ & Sn2-C8 & $2.143(3)$ \\
Sn1-Cl1 & $2.392(2)$ & Sn2-Cl2 & $2.403(2)$ \\
\hline
\end{tabular}

Data collection: APEX2 (Bruker, 2007); cell refinement: SAINT (Bruker, 2007); data reduction: $S A I N T$; program(s) used to solve structure: SHELXS97 (Sheldrick, 2008); program(s) used to refine structure: SHELXL97 (Sheldrick, 2008); molecular graphics: $X$ SEED (Barbour, 2001); software used to prepare material for publication: publCIF (Westrip, 2009).

The author thanks the University of Malaya for supporting this study.

Supplementary data and figures for this paper are available from the IUCr electronic archives (Reference: HB2901).

\title{
References
}

Barbour, L. J. (2001). J. Supramol. Chem. 1, 189-191.

Bruker (2007). APEX2 and SAINT. Bruker AXS Inc., Madison, Wisconsin, USA.

Flack, H. D. (1983). Acta Cryst. A39, 876-881.

Ng, S. W. (1997). Acta Cryst. C53, 56-58.

Sheldrick, G. M. (1996). SADABS. University of Göttingen, Germany.

Sheldrick, G. M. (2008). Acta Cryst. A64, 112-122.

Sisido, K., Takeda, Y. \& Kinugawa, Z. (1961). J. Am. Chem. Soc. 83, 538-541.

Westrip, S. P. (2009). publCIF. In preparation.

Yin, H.-D., Li, K.-Z. \& Hong, M. (2005). Huaxue Shiji, 27, 295-296, 310. 


\section{supporting information}

Acta Cryst. (2009). E65, m238 [doi:10.1107/S160053680900289X]

\section{Low-temperature redetermination of tribenzylchloridotin(IV)}

\section{Seik Weng Ng}

\section{S1. Comment}

The room-temperature structure of tribenzyltin(IV) chloride, (I), has been described in the $R 3$ space group but with the unique $c$-axis half as long $[\mathrm{a}=16.942(1), \mathrm{c}=5.9187(4) \AA](\mathrm{Ng}, 1997$; Yin et al., 2005) as that found here. Presumably, the two independent studies missed the weak reflections along the $c$-axis. In the present low-temperature study of (I) (Fig. 1 ), the $l=2 n+1$ reflections are generally weak but are unambiguously present. The crystal structure consists of $\left[\mathrm{SnCl}\left(\mathrm{C}_{7} \mathrm{H}_{7}\right)_{3}\right]$ molecules (Tabl 1) linked axially by tin $\cdots$ chlorine bridges into a chain along the $c$-axis of the trigonal unit cell.

\section{S2. Experimental}

Tribenzyltin chloride was prepared from metallic tin and benzyl chloride in water (Sisido et al., 1961) and was recrystallized from ethanol to yield colourless prisms of (I).

\section{S3. Refinement}

The $\mathrm{H}$ atoms were placed in calculated positions [C- $\left.\mathrm{C} 0.95-0.99 \AA, U_{\text {iso }}(\mathrm{H}) 1.2 U_{\text {eq }}(\mathrm{C})\right]$, and were included in the refinement in the riding-model approximation.

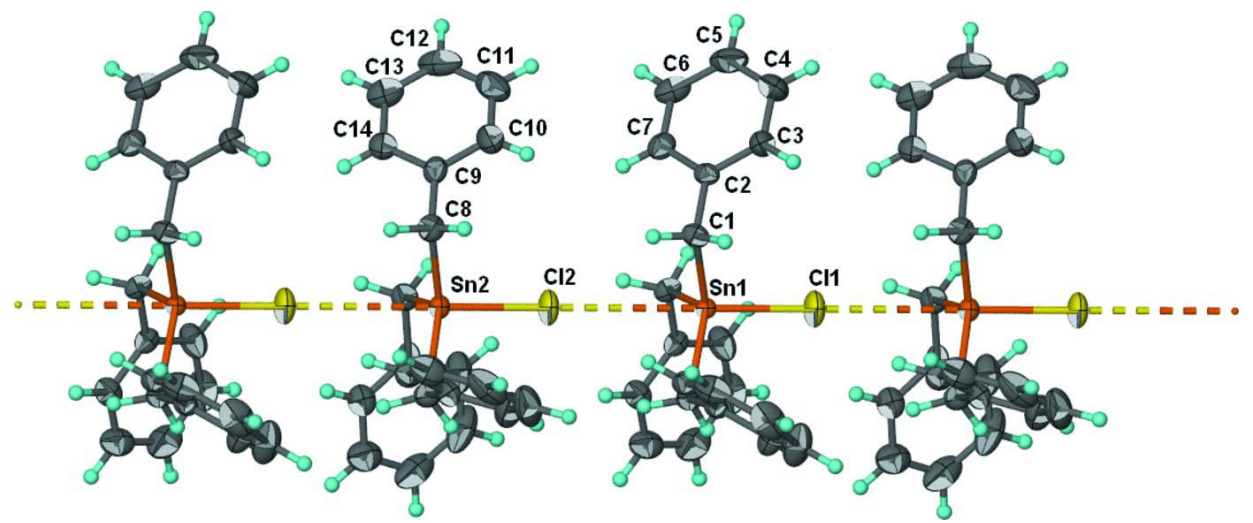

\section{Figure 1}

The molecular structure of (I); displacement ellipsoids are drawn at the $70 \%$ probability level, and $\mathrm{H}$ atoms as spheres of arbitrary radius. Only symmetry-independent atoms are labeled.

\section{tribenzylchloridotin(IV)}

Crystal data

$\left[\mathrm{Sn}\left(\mathrm{C}_{7} \mathrm{H}_{7}\right)_{3} \mathrm{Cl}\right]$

Trigonal, $R 3$

$M_{r}=427.52$

Hall symbol: R 3 
$a=16.7985$ (2) $\AA$

$c=11.6875(2) \AA$

$V=2856.23(6) \AA^{3}$

$Z=6$

$F(000)=1284$

$D_{\mathrm{x}}=1.491 \mathrm{Mg} \mathrm{m}^{-3}$

Mo $K \alpha$ radiation, $\lambda=0.71073 \AA$

Data collection

Bruker SMART APEX

diffractometer

Radiation source: fine-focus sealed tube

Graphite monochromator

$\omega$ scans

Absorption correction: multi-scan

(SADABS; Sheldrick, 1996)

$T_{\min }=0.589, T_{\max }=0.917$

\section{Refinement}

Refinement on $F^{2}$

Least-squares matrix: full

$R\left[F^{2}>2 \sigma\left(F^{2}\right)\right]=0.026$

$w R\left(F^{2}\right)=0.074$

$S=1.07$

2737 reflections

139 parameters

1 restraint

Primary atom site location: structure-invariant direct methods

Secondary atom site location: difference Fourier map
Cell parameters from 5172 reflections

$\theta=2.4-28.3^{\circ}$

$\mu=1.48 \mathrm{~mm}^{-1}$

$T=100 \mathrm{~K}$

Prism, colorless

$0.40 \times 0.08 \times 0.06 \mathrm{~mm}$

9077 measured reflections

2737 independent reflections

2431 reflections with $I>2 \sigma(I)$

$R_{\text {int }}=0.014$

$\theta_{\text {max }}=27.5^{\circ}, \theta_{\min }=2.2^{\circ}$

$h=-21 \rightarrow 21$

$k=-21 \rightarrow 21$

$l=-15 \rightarrow 14$

Hydrogen site location: inferred from neighbouring sites

$\mathrm{H}$-atom parameters constrained

$w=1 /\left[\sigma^{2}\left(F_{\mathrm{o}}^{2}\right)+(0.0515 P)^{2}+0.375 P\right]$

where $P=\left(F_{\mathrm{o}}{ }^{2}+2 F_{\mathrm{c}}{ }^{2}\right) / 3$

$(\Delta / \sigma)_{\max }=0.001$

$\Delta \rho_{\max }=0.53$ e $\AA^{-3}$

$\Delta \rho_{\min }=-0.25$ e $\AA^{-3}$

Absolute structure: Flack (1983), 1372 Fridel pairs

Absolute structure parameter: -0.01 (4)

Fractional atomic coordinates and isotropic or equivalent isotropic displacement parameters $\left(\AA^{2}\right)$

\begin{tabular}{lllll}
\hline & $x$ & $y$ & $z$ & $U_{\text {iso }} * / U_{\text {eq }}$ \\
\hline Sn1 & 0.3333 & 0.6667 & $0.500000(15)$ & $0.01587(10)$ \\
Sn2 & 0.3333 & 0.6667 & $1.00289(2)$ & $0.01945(10)$ \\
C11 & 0.3333 & 0.6667 & $0.29532(14)$ & $0.0388(4)$ \\
C12 & 0.3333 & 0.6667 & $0.79730(13)$ & $0.0364(4)$ \\
C1 & $0.4692(2)$ & $0.7751(2)$ & $0.5415(3)$ & $0.0231(6)$ \\
H1A & 0.4805 & 0.7730 & 0.6242 & $0.028^{*}$ \\
H1B & 0.5149 & 0.7659 & 0.4988 & $0.028^{*}$ \\
C2 & $0.4812(2)$ & $0.8672(2)$ & $0.5120(3)$ & $0.0214(6)$ \\
C3 & $0.5272(2)$ & $0.9129(2)$ & $0.4134(3)$ & $0.0353(7)$ \\
H3 & 0.5524 & 0.8858 & 0.3646 & $0.042^{*}$ \\
C4 & $0.5369(3)$ & $0.9971(2)$ & $0.3847(3)$ & $0.0475(9)$ \\
H4 & 0.5686 & 1.0272 & 0.3167 & $0.057^{*}$ \\
C5 & $0.5008(3)$ & $1.0378(2)$ & $0.4544(4)$ & $0.0438(8)$ \\
H5 & 0.5070 & 1.0954 & 0.4344 & $0.053^{*}$ \\
C6 & $0.4552(2)$ & $0.9929(2)$ & $0.5546(4)$ & $0.0361(8)$ \\
H6 & 0.4305 & 1.0204 & 0.6034 & $0.043^{*}$ \\
C7 & $0.4456(2)$ & $0.9089(2)$ & $0.5831(3)$ & $0.0283(7)$
\end{tabular}




\begin{tabular}{lllll} 
H7 & 0.4146 & 0.8791 & 0.6516 & $0.034^{*}$ \\
C8 & $0.4652(2)$ & $0.7841(2)$ & $1.0378(3)$ & $0.0269(7)$ \\
H8A & 0.4828 & 0.7815 & 1.1180 & $0.032^{*}$ \\
H8B & 0.5119 & 0.7831 & 0.9871 & $0.032^{*}$ \\
C9 & $0.4632(2)$ & $0.8710(2)$ & $1.0191(3)$ & $0.0259(6)$ \\
C10 & $0.4869(2)$ & $0.9179(2)$ & $0.9159(3)$ & $0.0417(8)$ \\
H10 & 0.5076 & 0.8957 & 0.8546 & $0.050^{*}$ \\
C11 & $0.4811(3)$ & $0.9968(2)$ & $0.9002(4)$ & $0.0531(10)$ \\
H11 & 0.4966 & 1.0271 & 0.8281 & $0.064^{*}$ \\
C12 & $0.4528(2)$ & $1.0317(2)$ & $0.9886(4)$ & $0.0482(9)$ \\
H12 & 0.4500 & 1.0863 & 0.9784 & $0.058^{*}$ \\
C13 & $0.4288(2)$ & $0.9860(2)$ & $1.0914(4)$ & $0.0401(8)$ \\
H13 & 0.4091 & 1.0091 & 1.1527 & $0.048^{*}$ \\
C14 & $0.4331(2)$ & $0.9067(2)$ & $1.1065(3)$ & $0.0311(7)$ \\
H14 & 0.4151 & 0.8756 & 1.1779 & $0.037^{*}$ \\
\hline
\end{tabular}

Atomic displacement parameters $\left(\AA^{2}\right)$

\begin{tabular}{lllllll}
\hline & $U^{11}$ & $U^{22}$ & $U^{33}$ & $U^{12}$ & $U^{13}$ & $U^{23}$ \\
\hline Sn1 & $0.01588(11)$ & $0.01588(11)$ & $0.01586(17)$ & $0.00794(5)$ & 0.000 & 0.000 \\
Sn2 & $0.02122(12)$ & $0.02122(12)$ & $0.01592(18)$ & $0.01061(6)$ & 0.000 & 0.000 \\
C11 & $0.0498(6)$ & $0.0498(6)$ & $0.0167(5)$ & $0.0249(3)$ & 0.000 & 0.000 \\
C12 & $0.0470(6)$ & $0.0470(6)$ & $0.0152(5)$ & $0.0235(3)$ & 0.000 & 0.000 \\
C1 & $0.0191(14)$ & $0.0214(14)$ & $0.0274(14)$ & $0.0092(11)$ & $-0.0005(11)$ & $0.0038(11)$ \\
C2 & $0.0192(13)$ & $0.0178(13)$ & $0.0233(13)$ & $0.0062(11)$ & $-0.0037(11)$ & $0.0006(10)$ \\
C3 & $0.0457(19)$ & $0.0255(15)$ & $0.0277(14)$ & $0.0125(14)$ & $0.0090(14)$ & $0.0008(11)$ \\
C4 & $0.070(2)$ & $0.0274(16)$ & $0.0344(19)$ & $0.0163(18)$ & $0.0067(16)$ & $0.0085(13)$ \\
C5 & $0.055(2)$ & $0.0231(15)$ & $0.050(2)$ & $0.0179(17)$ & $-0.0140(17)$ & $-0.0011(14)$ \\
C6 & $0.0319(18)$ & $0.0280(15)$ & $0.048(2)$ & $0.0150(14)$ & $-0.0070(14)$ & $-0.0085(14)$ \\
C7 & $0.0225(14)$ & $0.0270(15)$ & $0.0302(16)$ & $0.0086(12)$ & $-0.0012(11)$ & $-0.0059(12)$ \\
C8 & $0.0231(15)$ & $0.0284(16)$ & $0.0276(15)$ & $0.0116(13)$ & $0.0011(12)$ & $-0.0012(12)$ \\
C9 & $0.0246(14)$ & $0.0281(15)$ & $0.0208(12)$ & $0.0100(12)$ & $-0.0025(11)$ & $-0.0030(11)$ \\
C10 & $0.047(2)$ & $0.0328(17)$ & $0.0280(15)$ & $0.0066(16)$ & $0.0013(14)$ & $0.0007(13)$ \\
C11 & $0.063(2)$ & $0.0353(19)$ & $0.0365(19)$ & $0.0064(18)$ & $-0.0148(17)$ & $0.0104(15)$ \\
C12 & $0.049(2)$ & $0.0255(16)$ & $0.063(2)$ & $0.0138(17)$ & $-0.0256(18)$ & $-0.0023(16)$ \\
C13 & $0.0374(18)$ & $0.0338(18)$ & $0.050(2)$ & $0.0181(15)$ & $-0.0076(15)$ & $-0.0067(15)$ \\
C14 & $0.0300(16)$ & $0.0278(15)$ & $0.0299(16)$ & $0.0103(13)$ & $0.0012(12)$ & $-0.0008(12)$ \\
& & & & & & \\
\hline
\end{tabular}

Geometric parameters $\left(\hat{A},{ }^{o}\right)$

\begin{tabular}{llll}
\hline $\mathrm{Sn} 1-\mathrm{C} 1^{\mathrm{i}}$ & $2.146(3)$ & $\mathrm{C} 5-\mathrm{C} 6$ & $1.396(5)$ \\
$\mathrm{Sn} 1-\mathrm{C} 1$ & $2.146(3)$ & $\mathrm{C} 5-\mathrm{H} 5$ & 0.9500 \\
$\mathrm{Sn} 1-\mathrm{C} 1^{\mathrm{ii}}$ & $2.146(3)$ & $\mathrm{C} 6-\mathrm{C} 7$ & $1.379(5)$ \\
$\mathrm{Sn} 1-\mathrm{C} 11$ & $2.392(2)$ & $\mathrm{C} 6-\mathrm{H} 6$ & 0.9500 \\
$\mathrm{Sn} 1-\mathrm{C} 2$ & $3.475(2)$ & $\mathrm{C} 7-\mathrm{H} 7$ & 0.9500 \\
$\mathrm{Sn} 2-\mathrm{C} 8^{\mathrm{i}}$ & $2.143(3)$ & $\mathrm{C} 8-\mathrm{C} 9$ & $1.494(5)$ \\
$\mathrm{Sn} 2-\mathrm{C} 8$ & $2.143(3)$ & $\mathrm{C} 8-\mathrm{H} 8 \mathrm{~A}$ & 0.9900 \\
$\mathrm{Sn} 2-\mathrm{C} 8^{\mathrm{ii}}$ & $2.143(3)$ & $\mathrm{C} 8-\mathrm{H} 8 \mathrm{~B}$ & 0.9900
\end{tabular}




\begin{tabular}{|c|c|c|c|}
\hline $\mathrm{Sn} 2-\mathrm{Cl} 2$ & $2.403(2)$ & $\mathrm{C} 9-\mathrm{C} 10$ & $1.387(4)$ \\
\hline $\mathrm{Sn} 2-\mathrm{C} 11^{\mathrm{iii}}$ & $3.418(2)$ & $\mathrm{C} 9-\mathrm{C} 14$ & $1.400(4)$ \\
\hline $\mathrm{C} 1-\mathrm{C} 2$ & $1.497(4)$ & $\mathrm{C} 10-\mathrm{C} 11$ & $1.389(5)$ \\
\hline $\mathrm{C} 1-\mathrm{H} 1 \mathrm{~A}$ & 0.9900 & $\mathrm{C} 10-\mathrm{H} 10$ & 0.9500 \\
\hline $\mathrm{C} 1-\mathrm{H} 1 \mathrm{~B}$ & 0.9900 & $\mathrm{C} 11-\mathrm{C} 12$ & $1.383(5)$ \\
\hline $\mathrm{C} 2-\mathrm{C} 3$ & $1.387(4)$ & $\mathrm{C} 11-\mathrm{H} 11$ & 0.9500 \\
\hline $\mathrm{C} 2-\mathrm{C} 7$ & $1.398(4)$ & $\mathrm{C} 12-\mathrm{C} 13$ & $1.373(5)$ \\
\hline $\mathrm{C} 3-\mathrm{C} 4$ & $1.381(4)$ & $\mathrm{C} 12-\mathrm{H} 12$ & 0.9500 \\
\hline $\mathrm{C} 3-\mathrm{H} 3$ & 0.9500 & $\mathrm{C} 13-\mathrm{C} 14$ & $1.381(5)$ \\
\hline $\mathrm{C} 4-\mathrm{C} 5$ & $1.383(5)$ & $\mathrm{C} 13-\mathrm{H} 13$ & 0.9500 \\
\hline $\mathrm{C} 4-\mathrm{H} 4$ & 0.9500 & $\mathrm{C} 14-\mathrm{H} 14$ & 0.9500 \\
\hline $\mathrm{C} 11^{\mathrm{i}}-\mathrm{Sn} 1-\mathrm{C} 1$ & $115.06(6)$ & $\mathrm{C} 5-\mathrm{C} 4-\mathrm{H} 4$ & 119.8 \\
\hline $\mathrm{C} 1^{\mathrm{i}}-\mathrm{Sn} 1-\mathrm{C} 1^{\mathrm{ii}}$ & $115.06(7)$ & $\mathrm{C} 4-\mathrm{C} 5-\mathrm{C} 6$ & $119.0(3)$ \\
\hline $\mathrm{C} 1-\mathrm{Sn} 1-\mathrm{C} 1^{\mathrm{ii}}$ & $115.06(6)$ & $\mathrm{C} 4-\mathrm{C} 5-\mathrm{H} 5$ & 120.5 \\
\hline $\mathrm{C} 11^{\mathrm{i}}-\mathrm{Sn} 1-\mathrm{C} 11$ & $103.05(9)$ & $\mathrm{C} 6-\mathrm{C} 5-\mathrm{H} 5$ & 120.5 \\
\hline $\mathrm{C} 1-\mathrm{Sn} 1-\mathrm{C} 11$ & $103.05(9)$ & $\mathrm{C} 7-\mathrm{C} 6-\mathrm{C} 5$ & $120.5(3)$ \\
\hline $\mathrm{C} 1{ }^{\mathrm{ii}}-\mathrm{Sn} 1-\mathrm{C} 11$ & $103.05(9)$ & $\mathrm{C} 7-\mathrm{C} 6-\mathrm{H} 6$ & 119.8 \\
\hline $\mathrm{C} 1-\mathrm{Sn} 1-\mathrm{Cl} 2$ & $76.95(9)$ & $\mathrm{C} 5-\mathrm{C} 6-\mathrm{H} 6$ & 119.8 \\
\hline $\mathrm{C} 1-\mathrm{Sn} 1-\mathrm{Cl} 2$ & $76.95(9)$ & $\mathrm{C} 6-\mathrm{C} 7-\mathrm{C} 2$ & $120.6(3)$ \\
\hline $\mathrm{C} 1{ }^{\mathrm{ii}}-\mathrm{Sn} 1-\mathrm{Cl} 2$ & $76.95(9)$ & $\mathrm{C} 6-\mathrm{C} 7-\mathrm{H} 7$ & 119.7 \\
\hline $\mathrm{Cl} 1-\mathrm{Sn} 1-\mathrm{Cl} 2$ & 180.0 & $\mathrm{C} 2-\mathrm{C} 7-\mathrm{H} 7$ & 119.7 \\
\hline $\mathrm{C} 88^{\mathrm{i}}-\mathrm{Sn} 2-\mathrm{C} 8$ & $116.46(6)$ & $\mathrm{C} 9-\mathrm{C} 8-\mathrm{Sn} 2$ & $110.7(2)$ \\
\hline $\mathrm{C} 8^{\mathrm{i}}-\mathrm{Sn} 2-\mathrm{C} 8^{\mathrm{ii}}$ & $116.46(6)$ & $\mathrm{C} 9-\mathrm{C} 8-\mathrm{H} 8 \mathrm{~A}$ & 109.5 \\
\hline $\mathrm{C} 8-\mathrm{Sn} 2-\mathrm{C} 8^{\mathrm{ii}}$ & $116.46(6)$ & $\mathrm{Sn} 2-\mathrm{C} 8-\mathrm{H} 8 \mathrm{~A}$ & 109.5 \\
\hline $\mathrm{C} 8-\mathrm{Sn} 2-\mathrm{Cl} 2$ & $100.98(9)$ & $\mathrm{C} 9-\mathrm{C} 8-\mathrm{H} 8 \mathrm{~B}$ & 109.5 \\
\hline $\mathrm{C} 8-\mathrm{Sn} 2-\mathrm{Cl} 2$ & $100.98(9)$ & $\mathrm{Sn} 2-\mathrm{C} 8-\mathrm{H} 8 \mathrm{~B}$ & 109.5 \\
\hline $\mathrm{C} 8{ }^{\mathrm{ii}}-\mathrm{Sn} 2-\mathrm{Cl} 2$ & $100.98(9)$ & $\mathrm{H} 8 \mathrm{~A}-\mathrm{C} 8-\mathrm{H} 8 \mathrm{~B}$ & 108.1 \\
\hline $\mathrm{C} 8^{\mathrm{i}}-\mathrm{Sn} 2-\mathrm{C} 11^{\mathrm{iii}}$ & $79.02(9)$ & $\mathrm{C} 10-\mathrm{C} 9-\mathrm{C} 14$ & $117.0(3)$ \\
\hline $\mathrm{C} 8-\mathrm{Sn} 2-\mathrm{C} 11^{\mathrm{iii}}$ & $79.02(9)$ & $\mathrm{C} 10-\mathrm{C} 9-\mathrm{C} 8$ & $122.9(3)$ \\
\hline $\mathrm{C} 8^{\mathrm{ii}}-\mathrm{Sn} 2-\mathrm{C} 11^{\mathrm{iii}}$ & $79.02(9)$ & $\mathrm{C} 14-\mathrm{C} 9-\mathrm{C} 8$ & $120.1(3)$ \\
\hline $\mathrm{Cl} 2-\mathrm{Sn} 2-\mathrm{C} 11^{\mathrm{iii}}$ & 180.0 & $\mathrm{C} 9-\mathrm{C} 10-\mathrm{C} 11$ & $121.4(3)$ \\
\hline $\mathrm{Sn} 2-\mathrm{Cl} 2-\mathrm{Sn} 1$ & 180.0 & $\mathrm{C} 9-\mathrm{C} 10-\mathrm{H} 10$ & 119.3 \\
\hline $\mathrm{C} 2-\mathrm{C} 1-\mathrm{Sn} 1$ & $111.2(2)$ & $\mathrm{C} 11-\mathrm{C} 10-\mathrm{H} 10$ & 119.3 \\
\hline $\mathrm{C} 2-\mathrm{C} 1-\mathrm{H} 1 \mathrm{~A}$ & 109.4 & $\mathrm{C} 12-\mathrm{C} 11-\mathrm{C} 10$ & $120.5(3)$ \\
\hline $\mathrm{Sn} 1-\mathrm{C} 1-\mathrm{H} 1 \mathrm{~A}$ & 109.4 & $\mathrm{C} 12-\mathrm{C} 11-\mathrm{H} 11$ & 119.7 \\
\hline $\mathrm{C} 2-\mathrm{C} 1-\mathrm{H} 1 \mathrm{~B}$ & 109.4 & $\mathrm{C} 10-\mathrm{C} 11-\mathrm{H} 11$ & 119.7 \\
\hline $\mathrm{Sn} 1-\mathrm{C} 1-\mathrm{H} 1 \mathrm{~B}$ & 109.4 & $\mathrm{C} 13-\mathrm{C} 12-\mathrm{C} 11$ & $118.9(3)$ \\
\hline $\mathrm{H} 1 \mathrm{~A}-\mathrm{C} 1-\mathrm{H} 1 \mathrm{~B}$ & 108.0 & $\mathrm{C} 13-\mathrm{C} 12-\mathrm{H} 12$ & 120.5 \\
\hline $\mathrm{C} 3-\mathrm{C} 2-\mathrm{C} 7$ & $118.4(3)$ & $\mathrm{C} 11-\mathrm{C} 12-\mathrm{H} 12$ & 120.5 \\
\hline $\mathrm{C} 3-\mathrm{C} 2-\mathrm{C} 1$ & $120.9(3)$ & $\mathrm{C} 12-\mathrm{C} 13-\mathrm{C} 14$ & $120.6(3)$ \\
\hline $\mathrm{C} 7-\mathrm{C} 2-\mathrm{C} 1$ & $120.7(3)$ & $\mathrm{C} 12-\mathrm{C} 13-\mathrm{H} 13$ & 119.7 \\
\hline $\mathrm{C} 4-\mathrm{C} 3-\mathrm{C} 2$ & $121.1(3)$ & $\mathrm{C} 14-\mathrm{C} 13-\mathrm{H} 13$ & 119.7 \\
\hline $\mathrm{C} 4-\mathrm{C} 3-\mathrm{H} 3$ & 119.5 & $\mathrm{C} 13-\mathrm{C} 14-\mathrm{C} 9$ & $121.6(3)$ \\
\hline $\mathrm{C} 2-\mathrm{C} 3-\mathrm{H} 3$ & 119.5 & $\mathrm{C} 13-\mathrm{C} 14-\mathrm{H} 14$ & 119.2 \\
\hline $\mathrm{C} 3-\mathrm{C} 4-\mathrm{C} 5$ & $120.5(3)$ & $\mathrm{C} 9-\mathrm{C} 14-\mathrm{H} 14$ & 119.2 \\
\hline $\mathrm{C} 3-\mathrm{C} 4-\mathrm{H} 4$ & 119.8 & & \\
\hline
\end{tabular}




$\begin{array}{ll}\mathrm{C} 1{ }^{\mathrm{i}}-\mathrm{Sn} 1-\mathrm{C} 1-\mathrm{C} 2 & -41.4(3) \\ \mathrm{C} 1 \mathrm{i}-\mathrm{S} 1-\mathrm{C} 1-\mathrm{C} 2 & -178.70(16) \\ \mathrm{C} 11-\mathrm{S} 1-\mathrm{C} 1-\mathrm{C} 2 & 69.9(2) \\ \mathrm{C} 12-\mathrm{S} 1-\mathrm{C} 1-\mathrm{C} 2 & -110.1(2) \\ \mathrm{S} 1-\mathrm{C} 1-\mathrm{C} 2-\mathrm{C} 3 & -101.4(3) \\ \mathrm{S} 1-\mathrm{C} 1-\mathrm{C} 2-\mathrm{C} 7 & 78.1(3) \\ \mathrm{C} 7-\mathrm{C} 2-\mathrm{C} 3-\mathrm{C} 4 & -0.7(5) \\ \mathrm{C} 1-\mathrm{C} 2-\mathrm{C} 3-\mathrm{C} 4 & 178.8(3) \\ \mathrm{C} 2-\mathrm{C} 3-\mathrm{C} 4-\mathrm{C} 5 & 0.0(5) \\ \mathrm{C} 3-\mathrm{C} 4-\mathrm{C} 5-\mathrm{C} 6 & 0.6(6) \\ \mathrm{C} 4-\mathrm{C} 5-\mathrm{C} 6-\mathrm{C} 7 & -0.5(5) \\ \mathrm{C} 5-\mathrm{C} 6-\mathrm{C} 7-\mathrm{C} 2 & -0.2(5) \\ \mathrm{C} 3-\mathrm{C} 2-\mathrm{C} 7-\mathrm{C} 6 & 0.8(5) \\ \mathrm{C} 1-\mathrm{C} 2-\mathrm{C} 7-\mathrm{C} 6 & -178.7(3)\end{array}$

$\mathrm{C} 8-\mathrm{Sn} 2-\mathrm{C} 8-\mathrm{C} 9$
$\mathrm{C} 8{ }^{\mathrm{ii}}-\mathrm{Sn} 2-\mathrm{C} 8-\mathrm{C} 9$
$\mathrm{C} 12-\mathrm{S} 2-\mathrm{C} 8-\mathrm{C} 9$
$\mathrm{C} 11$ iii- 2 2 $-\mathrm{C} 8-\mathrm{C} 9$
$\mathrm{Sn} 2-\mathrm{C} 8-\mathrm{C} 9-\mathrm{C} 10$
$\mathrm{~S} 2-\mathrm{C} 8-\mathrm{C} 9-\mathrm{C} 14$
$\mathrm{C} 14-\mathrm{C} 9-\mathrm{C} 10-\mathrm{C} 11$
$\mathrm{C} 8-\mathrm{C} 9-\mathrm{C} 10-\mathrm{C} 11$
$\mathrm{C} 9-\mathrm{C} 10-\mathrm{C} 11-\mathrm{C} 12$
$\mathrm{C} 10-\mathrm{C} 11-\mathrm{C} 12-\mathrm{C} 13$
$\mathrm{C} 11-\mathrm{C} 12-\mathrm{C} 13-\mathrm{C} 14$
$\mathrm{C} 12-\mathrm{C} 13-\mathrm{C} 14-\mathrm{C} 9$
$\mathrm{C} 10-\mathrm{C} 9-\mathrm{C} 14-\mathrm{C} 13$
$\mathrm{C} 8-\mathrm{C} 9-\mathrm{C} 14-\mathrm{C} 13$

$-31.7(3)$

$-175.18(15)$

$76.6(2)$

$-103.4(2)$

-92.7 (3)

84.8 (3)

$0.1(5)$

$177.7(3)$

$1.2(5)$

$-1.3(5)$

$0.2(5)$

$1.1(5)$

$-1.2(5)$

$-178.9(3)$

Symmetry codes: (i) $-y+1, x-y+1, z$; (ii) $-x+y,-x+1, z$; (iii) $x, y, z+1$. 